\title{
Gallium nitrate and spinal cord injury. A pilot study of efficacy using an experimental model
}

\author{
S C Colachis MD,${ }^{1} \mathrm{P}$ G Popovich MS, ${ }^{2}$ B Stokes $\mathrm{PhD}^{3}$ \\ ${ }^{1}$ Assistant Professor, Department of Physical Medicine and Director, Spinal Cord Injury \\ Rehabilitation, ${ }^{2}$ Graduate Research Associate. The Department of Physiology, ${ }^{3}$ Professor, \\ Departments of Physiology and Surgery and Director, Spinal Cord Injury Research \\ Center, The Ohio State University, College of Medicine, Columbus, Ohio, USA.
}

Gallium ( $\mathrm{Ga}$ ) nitrate has been shown to attenuate immune-mediated tissue destruction in models of neurological autoimmunity. Based upon these observations, we evaluated the efficacy of Ga nitrate in attenuating the inflammatory response and modifying clinical recovery in an experimental model of traumatic spinal cord injury (SCI). Using a randomized, double-blind, placebo-controlled design, 12 adult female Spraque-Dawley rats received injections of either $\mathrm{Ga}$ nitrate $(n=8)$ or saline $(n=4)$ following experimental SCI. Ga treated animals received $30 \mathrm{mg} / \mathrm{kg}$ elemental $\mathrm{Ga}$ followed by weekly injections of $10 \mathrm{mg} / \mathrm{kg}$ elemental Ga. There were no observable differences between the Ga treated and the saline control groups using various behavioral paradigms examined during the study period. Histological and immunocytochemical data qualitatively showed no observable differences between $\mathrm{Ga}$ treated $(n=4)$ and control $(n=2)$ groups sacrificed 18 days following SCI. Although the efficacy of Ga in this pilot study of experimental SCI was not demonstrated, the potential of Ga to attenuate inflammatory-mediated reactions remains an exciting possibility in the area of SCI. Our preliminary results should not discourage future research endeavors in this regard. A more complete analysis of dose response, time and mode of $\mathrm{Ga}$ administration (preinjury or postinjury), and availability of $\mathrm{Ga}$ across the blood-brain barrier is needed to further evaluate the efficacy of this compound.

Keywords: spinal cord injury; gallium nitrate.

\section{Introduction}

Therapeutic interventions directed at minimizing spinal cord damage following an acute traumatic spinal cord injury (SCI) are based upon our understanding of the sequence of events occurring within minutes to hours of the neurologic insult. Disruption of vascular perfusion and extracellular ionic activity, followed by edema, metabolic derangements, and free radical reactions is concomitant with the initiation of an inflammatory process and release of excitatory amino acids. ${ }^{1.2}$ The concept of 'secondary

Correspondence: Sam C Colachis, MD The Ohio State University, Dodd Hall/Davis Center, 480 West Ninth Avenue, Columbus, Ohio 43210, USA. pathology ${ }^{2-4}$ involves this complex cascade of events following SCI which can result in progression of tissue ischemia and permanent destruction of injured neural elements.

Research efforts directed at modulating secondary spinal cord injury have involved the use of agents such as calcium channel blockers, ${ }^{5}$ antiadrenergic compounds, ${ }^{6}$ osmotic diuretics, ${ }^{7}$ enzyme therapy, ${ }^{8}$ local anesthetics, ${ }^{9}$ opiate antagonists, ${ }^{10}$ gangliosides, ${ }^{11}$ and steroids. ${ }^{10}$ The efficacy of highdose steroids following acute traumatic SCI in humans ${ }^{10}$ provides strong support for modulating the associated inflammatory process. ${ }^{12}$ Although secondary inflammation appears to play an integral role in the development of further spinal injury, ${ }^{2}$ the precise mechanisms have not been fully 
elucidated. Despite this, investigators have postulated a central role of the tissue macrophage in the secondary injury observed following experimentally induced $\mathrm{SCI} .^{2-4}$ Such observations give impetus to further evaluation of this immunologic interaction.

Gallium $(\mathrm{Ga})$ is a group IIIA transitional element with multiple valences which in one of its salt forms (ie $\mathrm{Ga}$ nitrate) can inhibit macrophage and lymphocyte activity under experimental conditions. ${ }^{13-15} \mathrm{Ga}$ nitrate has shown efficacy in suppression of experimental autoimmune encephalomyelitis, ${ }^{15}$ as well as in prevention of adjuvant arthritis $^{13.16}$ in rats. The use of Ga salts in the clinical treatment of Paget's disease may inhibit the function of osteoclasts, macrophage-like cells derived from bone marrow precursors. ${ }^{13.14}$ The present pilot study was designed to determine the efficacy of $\mathrm{Ga}$ nitrate in modifying SCI and spinal cord recovery in an experimental SCI model.

\section{Methods}

Animals

Twelve adult female Sprague-Dawley rats weighing between 250 and $275 \mathrm{~g}$ were utilized (Harlan Sprague-Dawley, Inc, Indianapolis, IN). All animals were housed in a controlled environment with regularly cycled light/dark periods of 12 hours. The animals were familiarized with their environment prior to the onset of the project, and allowed free access to food and water. Approval of the research protocol and treatment of the animals throughout the duration of the study was in accordance with the doctrines of the Animal Review Committee at The Ohio State University.

\section{Spinal cord impactor device}

Contusion lesions in the spinal cords of the animals were produced by our Ohio State impact device. ${ }^{17}$ This impact device delivers controlled displacement, force, and acceleration of the impactor probe, while allowing feedback of these parameters. ${ }^{17.18}$ Two highly sensitive transducers are attached to the impactor probe used to pro- duce experimental spinal cord injury in order to determine a known starting point for injury production, produce a known and reproducible displacement profile during the injury, and estimate the force needed to compress the spinal cord by the same amount for each animal. Such force feedback is quite useful in determining the biomechanical properties of the spinal compartment during the injury and in preventing the impactor probe from contacting adjacent tissue during the impact phase. It has shown previously that this impact device produces predictable incomplete spinal cord injuries of similar severity between animal groups. ${ }^{17-19}$

\section{Production of SCI lesions}

The animals were anesthetized with an intraperitoneal solution of ketamine hydrochloride $(80 \mathrm{mg} / \mathrm{kg})$ and xylazine $(10 \mathrm{mg} / \mathrm{kg})$. A partial dorsal laminectomy was then performed at T8-9, ensuring minimal disturbance of the dura. The laminectomy site was limited to one vertebral level, which was slightly larger than the tip of the impactor probe $\left(7 \mathrm{~mm}^{2}\right)$.

Following laminectomy, the animals were mounted in a spinal frame in which the nearby dorsal processes were rigidly fixed, as described previously. ${ }^{19-21}$ A Ling shaker (Model V203/S, Ling Dynamics Systems Corp) was used to compress the spinal cord dorsal surface approximately $1.1 \mathrm{~mm}$ over a $23 \mathrm{~ms}$ time period. The injury process involves approximately $11 \mathrm{~ms}$ to produce the full impact and reach peak compression, followed by removal of the impactor probe from contact with the dural surface of the cord over the remaining $12 \mathrm{~ms}$ time period.

Muscle and skin wounds were then closed in layers. Following surgery, the animals were placed on a heating pad and allowed to recover spontaneously. They were given fluids to maintain hydration and were allowed to eat ad libitum as they recovered. Bladders were expressed at least three times daily until reflexes returned (usually within 2 weeks). Antibiotics were administered orally ( $4.0 \mathrm{mg}$ trimethoprim; $20 \mathrm{mg}$ sulfadiazine, daily) from 2 days preoperatively until reflex bladder function returned. 


\section{Gallium nitrate injection protocol}

Rats were randomized into two groups to receive either $\mathrm{Ga}$ nitrate $(n=8)$ or saline $(n=4)$. Ga nitrate (Ben Venue Laboratories, Inc, Bedford, Ohio) was a gift from the National Cancer Institute (Bethesda, Maryland). Ga nitrate was administered to the rats subcutaneously in the nape of the neck at 1 hour after spinal cord injury as an initial bolus of $30 \mathrm{mg} / \mathrm{kg}$ of elemental $\mathrm{Ga}$. Thereafter, the animals received weekly subcutaneous injections of $\mathrm{Ga}$ nitrate in the nape of the neck $(10 \mathrm{mg} / \mathrm{kg}$ of elemental $\mathrm{Ga}$ ) for the following 2 weeks. The follow up injections required the use of a restraint cage to ensure that the complete dose was given. The dosing schedule was based upon prior experimental studies demonstrating the clinical efficacy of $\mathrm{Ga}$ nitrate in rodent models of experimental adjuvant arthritis ${ }^{13}$ and experimental autoimmune encephalomyelitis. ${ }^{15}$ All investigators and laboratory personnel involved in this study were blinded from knowledge of which animals received $\mathrm{Ga}$ nitrate or saline solution.

\section{Evaluation of general motor functions}

Each of the animals was conditioned to three different paradigms (ie Tarlov openfield scoring, inclined plane analysis, and footfalls on an open grid) for several weeks prior to receiving a spinal cord injury. All animals were scored on the behavioral trials on at least two separate occasions prior to injury. The animals were followed after spinal cord injury by repeated longitudinal assessments of generalized motor behaviors. They were tested on days 0 (before SCI), 1 , $2,3,8,14,18$, and 28 following SCI; six animals were sacrificed 18 days after injury, leaving the six remaining animals to be tested 28 days after injury.

We used the Tarlov open-field scoring, ${ }^{22}$ inclined plane analysis, ${ }^{23}$ and footfalls on an open $\operatorname{grid}^{20.24}$ in this pilot study. The Tarlov open-field scoring ${ }^{21.22}$ is a rating scale for general over-ground locomotion that has a high interrater reliability. ${ }^{21}$ The limbs of the animals were individually scored and averaged for each trial and day of analysis. The inclined plane test allowed for a general test of behavioral compensation for displace- ment in space. Animals were tested for 5 seconds in each of three positions: head up, right side up, or left side up. Animals respond consistently over chronic postinjury times. The grid walking task ${ }^{20.24}$ assessed the animals' ability to traverse a wire mesh with widely spaced bars $(3.7 \mathrm{~cm}$ apart $)$. Animals were required to move for at least 30 seconds on this device in a 3-minute period while the footfalls of the hind feet through the wire mesh were counted (in number of footfalls per second).

These behavioral tasks have been shown in our previous studies of contusion injuries to correlate highly with biomechanical injury descriptors, lesion geometry, and physiological analysis of the injury process. ${ }^{19} \cdot 21.2+$ Each has unique predictive value during different times after injury. ${ }^{17.18 .21}$

\section{Tissue collection, histology, and}

immunocytochemistry

Six animals (four Ga, two saline treated) were sacrificed under deep anesthesia 18 days following SCI, and their spinal cords were removed using the method of De Sousa and Horrocks. ${ }^{25}$ Application of hydraulic pressure via syringe to the caudal spinal canal forced the intact spinal cord through a cervical opening created by decapitation. The spinal cord specimens were then quickly frozen in liquid nitrogen. Serial coronal sections were cut on a cryostat $(20$ micron thickness); sections extended from $5 \mathrm{~mm}$ rostral to the spinal cord lesion center, and through the lesion epicenter, to $5 \mathrm{~mm}$ caudal. The sections were collected on gelatin coated slides and stored at $-20^{\circ} \mathrm{C}$.

The tissue sections were stained with the OX-6 monoclonal antibody (Bioproducts for Science, Inc, Indianapolis, IN), which is specific for the monomorphic determinant of the major histocompatibility complex (MHC) class II antigen. ${ }^{26}$ The primary antibody was diluted to a working dilution of 1:500. Antibody binding was visualized using preabsorbed biotinylated secondary antibody (Vector Labs, CA) and streptavidin conjugated horseradish peroxidase (HRP) (Zymed, CA), followed by reaction with 3,3'-diaminobenzidine- $\mathrm{H}_{2} \mathrm{O}_{2}$ substrate medium. Omission of primary antibody 
served as a control. Several sections were counterstained with cresyl violet or neutral red to visualize gliosis and mass inflammatory infiltrate. Myelin profiles of adjacent sections were visualized using luxol fast blue (LFB) staining. Qualitative assessments of gliotic scarring, mass inflammatory infiltrate, and myeline profiles were performed by direct visualization.

\section{Statistical analysis}

A two dimensional analysis of variance (ANOVA) was performed on mean behavioral values between treatment and control groups for the various paradigms utilized in the study.

\section{Results}

Eight rats received $\mathrm{Ga}$ nitrate injections and four received normal saline. There were no differences observed between the $\mathrm{Ga}$ treated and the saline control groups using the behavioral paradigms examined during the study period (ie 28 days postinjury). The Tarlov open-field test scores, average inclined plane scores, and average grid walking scores are depicted in Figures 1a-c. $P$ values of less than 0.05 were considered significant.

Histological and immunocytochemical data similarly showed no qualitatively observable differences between $\mathrm{Ga}$ treated $(n=4)$ and control $(n=2)$ groups sacrificed 18 days following SCI. We observed MHC class II antigen reaction on both macrophages and microglia in the injured spinal cord tissues which was qualitatively indistinguishable between control and $\mathrm{Ga}$ treated animals. Gliotic scarring and inflammatory cell density were also indistinguishable between groups.

\section{Discussion}

$\mathrm{Ga}$ is a group IIIA transitional element clinically used in the diagnosis of suspected neoplastic ${ }^{27}$ and inflammatory conditions, ${ }^{28}$ as well as in the treatment of hypercalcemia associated with malignancy. ${ }^{29-31}$ This metal is reported to have efficacy in the treatment of certain neoplasms, ${ }^{29.32}$ and has profound
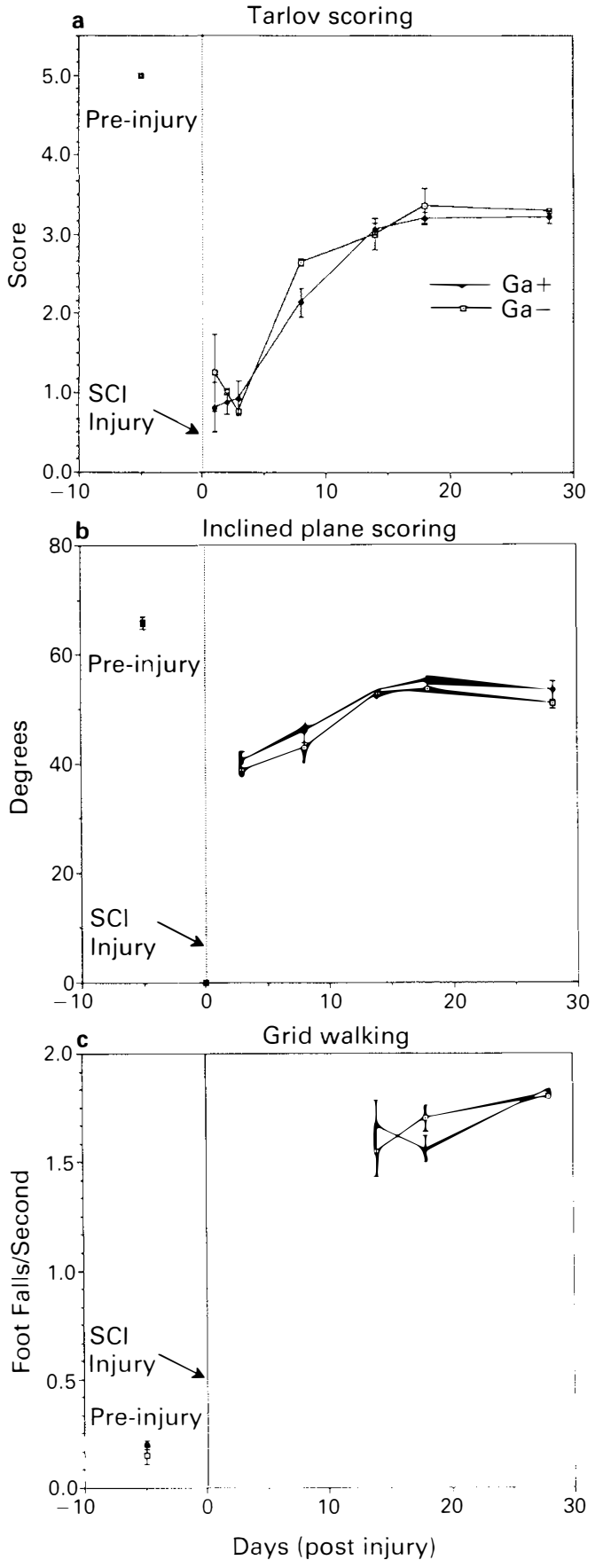

Figure 1 Behavioral paradigms used in evaluating neurologic recovery following SCI in gallium treated $(\mathrm{Ga}+)$ and saline control $(\mathrm{Ga}-)$ animals. (a) Tarlov scoring. (b) Inclined plane scoring. (c) Grid walking. (The error bars represent standard error of the mean.) 
effects upon bone metabolism. ${ }^{33-36}$ In addition to its clinical applications, $\mathrm{Ga}$ appears to have immunologic activity. ${ }^{13.15} \cdot 16.37$

In the current pilot study, we postulated that $\mathrm{Ga}$ nitrate, by suppressing macrophage function, could modulate the inflammatory sequelae of acute traumatic SCI and improve neurologic recovery in the experimental model. Blight ${ }^{2-4.38}$ has suggested that the macrophage could be a key cellular component in the process of secondary injury following acute traumatic SCI. Correlations of hypervascularity with declining neurologic scores and the delayed onset of functional loss in animals following administration of a macrophage toxin, support this hypothesis.

Our initial attempts to use $\mathrm{Ga}$ nitrate as an immunosuppressive agent following experimental SCI in rodents have met with negative results. Using three behavioral paradigms, we were unable to observe any significant differences in recovery of spinal cord function following spinal injury. Similarly, no significant histologic differences were observed. The expression of MHC class II antigen from microglia and macrophages observed in our SCI model signifies a state of activation. ${ }^{39} \mathrm{MHC}$ class II antigen is normally not expressed in the rat central nervous system by these cells except in the presence of injury or inflammatory stimuli. Microglial and macrophage activation as determined by antibody visualization of MHC class II antigen was indistinguishable between the $\mathrm{Ga}$ treated and control groups.

These findings differed from our initial hypothesis and were unexpected given that $\mathrm{Ga}$ indeed appears to interfere with the function of both macrophages ${ }^{13.14}$ and lymphocytes. ${ }^{13,15.37}$ Matkovic et al ${ }^{13}$ observed that rats treated with $\mathrm{Ga}$ exhibited less prominent clinical and histopathological features of experimental adjuvant arthritis (induced by injection of complete Freund's adjuvant) compared with animals without $\mathrm{Ga}$ administration. They demonstrated an in vitro reduction in MHC class II antigen expression on macrophages treated with $\mathrm{Ga}$ nitrate: Ga also suppressed mitogenic and antigen-specific proliferative responses in a purified-protein-derivative-specific $\mathrm{T}$-cell line produced from Lewis rats. ${ }^{13}$ Whitacre et $a l^{15}$ recently showed suppression of clinical signs and histopathological findings of experimental autoimmune encephalomyelitis (EAE) by Ga nitrate administered subcutaneously in dosages similar to those given in our study. Using a purified myelin basic protein (MBP)-specific $\mathrm{T}$-cell line, they found that $\mathrm{Ga}$ affects a relatively early stage of T-cell activation. ${ }^{15}$ The effects of $\mathrm{Ga}$ on macrophage function in the EAE model are currently under investigation. ${ }^{15} \mathrm{Ga}$ has also been shown to have an inhibitory effect on the proliferation of $\mathrm{B}$ lymphocytes. ${ }^{37}$ The use of $\mathrm{Ga}$ nitrate appears to interfere with the function of osteoclasts, macrophage-like cells of bone marrow origin. ${ }^{14.34 .35}$ A Gallium-transferrin complex may inhibit ribonucleotide reductase, ${ }^{4(1-+2}$ interfere with DNA synthesis, ${ }^{40-42}$ and suppress the proliferation of osteoblasts and the function of osteoclasts. In vitro studies of macrophages have shown high levels of transferrin mediated $\mathrm{Ga}^{67}$ uptake. ${ }^{+3}$

Based upon the findings in the present pilot study, it is difficult to ascertain whether $\mathrm{Ga}$ nitrate has any therapeutic value in the treatment of acute traumatic SCI. The cascade of events involved in the acute inflammatory reaction following traumatic SCI are multiple (and nonexclusive) $)^{3}$ and inhibition of any aspect(s) of that process by $\mathrm{Ga}$ nitrate may not be significant enough to impart a notable clinical response. However, before reaching a conclusion regarding the efficacy of $\mathrm{Ga}$, several caveats of the present study must be addressed.

We administered $\mathrm{Ga}$ nitrate in dosing schedules similar to those used in other studies demonstrating the clinical efficacy of Ga nitrate in rodent models of experimental adjuvant arthritis ${ }^{13}$ and experimental autoimmune encephalomyelitis. ${ }^{15}$ Perhaps different dosages and/or dosage schedules of $\mathrm{Ga}$ nitrate than those employed are needed to observe a significant therapeutic response. It is unclear as to whether $\mathrm{Ga}$ crosses the blood-brain barrier (BBB) in uninjured animals, and to what extent it crosses following SCI. In order to determine the extent to which Ga enters uninjured or injured CNS parenchymal tissue, Ga conjugated to fluorescent or peroxidase tracers, 
or radiolabeled $\mathrm{Ga}^{67}$ could be utilized. In our model of SCI, we are uncertain of the duration of time during which the permeability to the $\mathrm{BBB}$ is altered, and assessment of $\mathrm{BBB}$ permeability over time would be helpful.

Studies on gallium's ability to attenuate macrophage function have thus far been limited to macrophages of bone marrow origin. At present, a controversy still exists as to the origin of the CNS microglia cells. The possibility that microglia are of a mesodermal origin as opposed to being formed from bone marrow precursors, specifically infiltrating monocytes, suggests that certain membrane receptor mechanisms necessary for Ga uptake may not be present on resident microglial cells. Therefore, microglial mediated cytotoxicity may not be affected by $\mathrm{Ga}$. Since the exact mechanism with which Ga exerts its effects are not fully elucidated, further in vitro studies would be helpful in characterizing the effect of $\mathrm{Ga}$ on microglial cells.

Although the efficacy of $\mathrm{Ga}$ in the present study of experimental SCI was not demonstrated, the potential of $\mathrm{Ga}$ to attenuate inflammatory-mediated reactions remains an exciting possibility in the area of SCI. These preliminary results hopefully will not discourage future research endeavors in this regard.

\section{References}

1 Young W (1993) Strategies for the development of new and better pharmacological treatments for acute spinal cord injury. Adv Neurol 59: 249-256.

2 Blight AR (1993) Remyelination, revascularization, and recovery of function in experimental spinal cord injury. Adv' Neurol 59: 91-104.

3 Blight AR (1991) Morphometric analysis of a model of spinal cord injury in guinea pigs, with behavioral evidence of delayed secondary pathology. J Neurol Sci 103: 156-171.

4 Blight AR (1985) Delayed demyelination and macrophage invasion: a candidate for secondary cell damage in spinal cord injury. CNS Trauma 2: 299-315.

5 Ford RWJ, Malm DN (1985) Failure of nimodipine to reverse acute experimental spinal cord injury. CNS Trauma 2: 9-16.

6 Hedeman LS, Sil R (1974) Studies in experimental spinal cord trauma. Part 2. Comparison treatment with steroids, low-molecular weight dextran and catecholamine blockage. J Neurosurg 40: 44-51.

7 De La Torre JC, Johnson CM, Goode DJ, Mullan S (1975) Pharmacologic treatment and evaluation of permanent experimental spinal cord trauma. Netrology (Minneapolis) 25: 508-514.

8 Guth L, Albuquerque EX, Deshpande SS, Barrett CP. Donati EJ, Warnick JE (1980) Ineffectiveness of enzyme therapy on regeneration in the transected spinal cord of the rat. J Neurosurg 52: 73-86.

9 Ford RWJ, Malm DN (1984) Failure of tetracaine to reverse spinal cord injury in the cat. J Neurosurg 60: $1269-1274$

10 Bracken MB, Shepard MJ, Collins WF, Holford TR, Young W, Baskin DS et al (1990) A randomized, controlled trial of methylprednisolone or naloxone in the treatment of acute spinal-cord injury: results of the Second National Acute Spinal Cord Injury Study. N Engl J Med 322: 1405-1411.

11 Geisler FH, Dorsey FC, Coleman WP (1991) Recovery of motor function after spinal-cord injury-A randomized, placebo-controlled trial with GM-1 ganglioside. N Engl J Med 324: 1829-1838.

12 Hsu CY, Dimitrijevic MR (1990) Methylprednisolone in spinal cord injury: the possible mechanism of action. J Neurotrauma 7: 115-119.

13 Matkovic V, Balboa A, Clinchot D. Whitacre C. Zwilling B, Brown D et al (1991) Gallium prevents adjuvant arthritis in rats and interferes with macrophage/T-cell function in the immune response. Curr Ther Res 50: 255-267.

14 Matkovic V. Gerber N, Brown D, Apseloff G, Zwilling BS (1991) Differential effect of gallium on MHC Class II expression by murine macrophages. FASEB J A1206: 4845.

15 Whitacre C, Apseloff G, Cox K, Matkovic V, Jewell S, Gerber N (1992) Suppression of experimental autoimmune encephalomyelitis by gallium nitrate. J Neuroimmunol 39: 175-182.

16 Collery Ph, Rinjard P, Pechery C (1990) Effect of gallium chloride on inflammation and experimental polyarthritis. In: Collery $\mathrm{Ph}$, Poirier LA, Manfait M, Etienne JC, editors. Metal Ions in Biology and Medicine. John Libbey Eurotext, Paris: 443-444.

17 Stokes BT, Noyes DH, Behrmann DL (1992) An electromechanical spinal injury device with dynamic sensitivity. J Neurotrauma 9: 187-195.

18 Beattie MS, Stokes BT, Bresnahan JC (1988) Experimental spinal cord injury: strategies for acute and chronic intervention based on anatomic, physiologic, and behavioral studies. In: Stein DG, Sabel BA, editors. Pharmacological Approaches to the Treatment of Brain and Spinal Cord Injury. Plenum Publishing Corp, New York: 43-74. 
19 Stokes BT, Reier PJ (1992) Fetal grafts alter chronic behavioral outcome after contusion damage to the adult rat spinal cord. Exp Neurol 116: 1-12.

20 Bresnahan JC, Beattie MS. Stokes BT. Conway KM (1991) Three-dimensional computer-assisted analysis of graded contusion lesions in the spinal cord of the rat. J Neurotrauma 8: 91-101.

21 Bresnahan JC. Beattie MS. Todd F, Noyes DH (1987) A behavioral and anatomical analysis of spinal cord injury produced by a feedback-controlled impaction device. Exp Neurol 95: 548-570.

22 Tarlov I (1957) Spinal Cord Compression: Mechanisms of Paralysis and Treatment. Charles C Thomas. Springfield, IL.

23 Rivlin A. Tator C (1977) Objective clinical assessment of motor function after experimental spinal cord injury in the rat. $J$ Neurosurg 47: 577-581.

24 Somerson SK. Stokes BT (1987) Functional analysis of an electromechanical spinal cord injury device. Exp Neurol 96: 82-96.

25 De Sousa BN, Horrocks LA (1979) Development of rat spinal cord. 1. Weight and length with a method for rapid removal. Dev Neurosci 2: 115-121.

26 McMaster WR. Williams AF (1979) Identification of Ia glycoproteins in rat thymus and purification from rat spleen. Eur J Immunol 9: 426-433.

27 Johnson GS (1981) Clinical applications of gallium in oncology. Int J Nucl Med Biol 8: 249-255.

28 Hoffer PB (1981) Use of gallium-67 for detection of inflammatory diseases. Int J Nucl Med Biol 8: 243-247.

29 Foster BJ, Clagett-Carr K. Hoth D. Leyland-Jones B (1986) Gallium nitrate: the second metal with clinical activity. Cancer Treat Rep 70: 1311-1319.

30 Warrell RP. Isreal R. Frisone M. Snyder T. Gavnor JJ. Bockman RS (1988) Gallium nitrate for acute treatment of cancer-related hypercalcemia: a randomized. double-blind comparison to calcitonin. Ann Intern Med 108: 669-674.

31 Warrell RP. Skelos A. Alcock NW. Bockman RS (1986) Gallium nitrate for acute treatment of cancerrelated hypercalcemia: clinicopharmacological and dose response analysis. Cancer Res 46: 4208-4212.

32 Kelsen DP. Alcock N. Yeh S. Brown J. Young C (1980) Pharmacokinetics of gallium nitrate in man. Cancer 46: 2009-2013.

33 Bockman R. Adelman R. Donnelly R. Brody L. Warrell R. Jones K (1990) Gallium a unique anti-resorptive agent in bone: pre-clinical studies on its mechanisms of action. In: Collery Ph. Poirer LA. Manfait M, Etienne JC. editors. Metal Ions in Biology and Medicine. John Libbey Eurotext. Paris: 426-431.

34 Hall TJ, Chambers TJ (1990) Gallium inhibits bone resorption by a direct effect on osteoclasts. Bone Mineral 8: 211-216.

35 Matkovic V. Apseloff G. Shepard DR. Gerber N (1990) Use of gallium to treat Paget's disease of bone: A pilot study. Lancet 335: $72-75$.

36 Warrell RP. Alcock NW. Bockman RS (1987) Gallium nitrate inhibits accelerated bone turnover in patients with bone metastases. J Clin Oncol 5: 292-298.

37 Kovar J, Seligman P. Gelfand EW (1990) Differential growth-inhibitory effects of gallium on B-lymphocyte lines in high versus low iron concentration. Cancer Res 50: 5727-5730.

38 Blight AR (1991) Morphometric analysis of blood vessels in chronic experimental spinal cord injury: hypervascularity and recovery of function. J Neurol Sci 106: 158-174.

39 Streit WJ, Graeber MB, Kreutzberg GW (1988) Functional plasticity of microglia: a review. Glia 1: 301-307.

40 Chitamber CR, Matthaeus WG, Antholine WE. Graff K. O`Brien WJ (1988) Inhibition of leukemic HL60) cell growth by transferrin-gallium: effects on ribonucleotide reductase and demonstration of drug synergy with hydroxyurea. Blood 72: 1930-1936.

41 Hedley DW, Tripp EH, Slowiaczek P. Mann GJ (1988) Effect of gallium on DNA synthesis by human T-cell lymphoblasts. Cancer Res 48: 3014-3018.

42 Larson SM, Rasey JS, Allen DR, Nelson NJ. Grunbaum Z. Harp GD. Williams DL (1980) Common pathway for tumor cell uptake of Ga-67 and Fe-59 via a transferrin receptor. J Natl Cancer Inst 64: 41-53.

43 Andreesen R, Sephton RG, Gadd S, Atkins RC. De Abrew S (1988) Human macrophage maturation in vitro: expression of functional transferrin binding sites of high affinity. Blut 57: 77-83. 